\title{
Interférences
}

Ars scribendi

$6 \mid 2012$

La question de la littérarité

\section{Térence et Ménandre selon Aelius Donatus}

Le grammairien Donat au premier niveau d'une critique littéraire

\section{Gualtiero Calboli}

\section{(2) OpenEdition}

Journals

Édition électronique

URL : http://journals.openedition.org/interferences/402

DOI : $10.4000 /$ interferences. 402

ISSN : $1777-5485$

Éditeur

HiSoMA - Histoire et sources des Mondes antiques

Édition imprimée

Date de publication : 1 janvier 2012

ISSN : 1777-5485

Référence électronique

Gualtiero Calboli, «Térence et Ménandre selon Aelius Donatus », Interférences [En ligne], 6 | 2012, mis en ligne le 16 janvier 2018, consulté le 15 septembre 2020. URL : http://journals.openedition.org/ interferences/402 ; DOI : https://doi.org/10.4000/interferences.402

Ce document a été généré automatiquement le 15 septembre 2020.

Tous droits réservés 


\title{
Térence et Ménandre selon Aelius Donatus
}

\author{
Le grammairien Donat au premier niveau d'une critique littéraire
}

Gualtiero Calboli

1 On sait bien que les plus grands spécialistes de poésie étaient, dans la tradition scolaire grecque et latine, les grammairiens, parce que l'éducation des jeunes était menée avec la lecture des poètes et que c'était là l'œuvre du grammairien. Aelius Donatus a été l'un des plus grands grammairiens du Bas Empire, commentateur de Virgile et de Térence, maître de saint Jérôme comme de son adversaire Rufin Wessner ${ }^{1}$.

2 On peut voir le grammairien Donat à l'œuvre dans son commentaire de Térence. Pour ma part, je le connaissais pour ses interventions sur le texte de Térence, où il le compare systématiquement à Ménandre, et j'ai pensé qu'il pouvait être intéressant d'étudier quelques passages de Térence dont Donat a donné un commentaire, surtout s'ils sont essentiels pour sa comédie.

3 Aussi bien Rieth ${ }^{2}$ que Büchner ${ }^{3}$ ont mis en évidence que, dans les Adelphes de Térence, au vers 940, Eschine appuie avec un mensonge l'invitation de son père naturel Déméa, adressée à son père d'adoption Micion, à se marier avec Sostrata, la mère veuve de la jeune Pamphila qu'Eschine lui-même va épouser :

(1) Ter., Ad. 937-946

MI. sătĭn sánŭs es? iamb.oct.

ĕgŏ nŏvŏ' măritŭs anno demum quinto et sexagesŭmo

fiam atque ănum decrěpitam ducam ? idne estǐs auctorés mìhi ?

$A E$. fac< $<\breve{e}>$ : promisi ĕgo illis. MI. promisti autem ? de te largitór, pŭer.

$D E$. ăğ, quid siquid te maiŭs oret? MI. quăsĭ non hoc sit maxŭmum.

$D E$. da věnĭam. AE. ne grăvarĕ. DE. fac, promitte. MI. non ŏmittĭtis?

AE. non, nissi te exōrem. MI. vis est haec quĭdem. DE. ăgĕ prolixe, Mič̌o.

MI. etsi hoc mihi pravom ineptum absurdum atque ălienum a vitá měa

vĭdetur, si vos tanto ŏpěre istuc volti', fiat. AE. běnĕ făcis.

měrĭtó te ămo.

MI. Es-tu dans ton bon sens? Que moi je fasse le jeune marié avec mes soixante-cinq ans, et que j'épouse une vieille décrépite? Voilà ce que vous me conseillez? ES.

Fais-le; je le leur ai promis. MI. Voire! tu as promis ? Généreux à ton compte, mon 
garçon! DÉ. Voyons, que serait-ce, s'il te demandait quelque chose de plus grave? MI. Comme si ce n'était pas là la plus grave qui soit! DÉ. Donne ton consentement! ES. Ne fais pas le difficile! DÉ. Va, promets! MI. Vous n'allez pas me lâcher? ES. Non, pas sans t'avoir fléchi. MI. Mais c'est là de la violence! DÉ. Allons, un bon mouvement, Micion! MI. Eh bien, encore que je trouve cela insensé, inepte, absurde, étranger à mon genre d'existence, si vous le souhaitez tellement, que cela soit ! ES. Tu es bien. Tu mérites l'amour que j'ai pour toi (trad. J. Marouzeau, Térence , III, CUF, p. 177 sq.).

De cette façon les deux familles seront unifiées comme les deux maisons de Micion et de Sostrata qui n'en feront qu'une, ce qui arrive vraiment à la fin de la pièce (v. 908-922). En effet, Déméa voulait prendre sa revanche sur son frère Micion, qui avait adopté l'un de ses deux fils, Eschine, l'autre s'appelant Ctésiphon. Déméa voulait donc prendre sa revanche sur le fait que l'éducation libérale de Micion avait abouti à de bons résultats, tandis que l'éducation rigide et campagnarde impartie à Ctésiphon par Déméa lui-même en avait obtenu de mauvais : Eschine était tombé amoureux d'une jeune fille d'Attique, quoique pauvre, son frère Ctésiphon d'une courtisane, Bacchis, esclave du leno Sannion. À ce moment-là précisément, celui-ci avait pris la décision d'aller la vendre au grand marché de Chypre, avec ses autres esclaves. Le tropos de la comédie offrait alors à Ctésiphon deux solutions désespérées : se suicider, ou aller à l'étranger servir comme soldat quelque roi hellénistique. On prendra garde au fait que de l'étranger on pouvait rentrer, ainsi Clinia dans l'Heautontimoroumenos ${ }^{4}$. Comme on verra, dans les Adelphes de Térence Ctésiphon menace de faire l'une et l'autre chose, et cela est singulier. Mais son frère Eschine est intervenu : il a enlevé la belle de la maison du leno Sannion et l'a emportée dans celle de Micion, qui est aussi la sienne. La comédie s'ouvre (et c'est le premier acte ${ }^{5}$, tiré des A $\mathrm{A} \delta \lambda \hat{\lambda}$ oí $\beta$ de Ménandre comme toute la comédie, à l'exception de l'acte II) avec le père adoptif Micion qui est en peine parce que Eschine n'est pas rentré chez lui la nuit précédente. En effet, il est allé enlever Bacchis de la maison du leno Sannion, mais pour son frère, et le premier acte, ou ce qui pourrait correspondre au premier acte, se termine par un monologue de Micion,

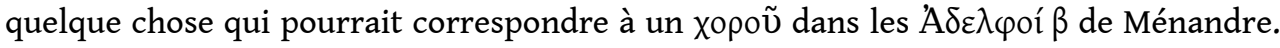
Après cette partie commence le locus tiré des $\Sigma u v \alpha \pi \circ \theta v \eta ́ \sigma \kappa o v \tau \varepsilon \varsigma$ de Diphile, et précisément du début de cette comédie. C'est une notice que Térence lui-même nous donne :
(2) Ter., Ad. 6-14
Synăpŏthnescontes Diphìli comoedĭast :
iamb.sen.
eam Commŏrientis Plautu' fecit fabŭlam.
in Graeca ădŭlescens est qui lenoni erĭpit
mĕrĕtricem in prima fabŭla : eum Plautús lŏcum
rěliquĭt intěgrum, eum hic lŏcum sumpsít sibi
in Ădelphos, verbum de verbo expressum extŭlit,
eam nos acturi sŭmŭ' nŏvam : pernoscite
furtumnĕ factum existŭmeť̌s an lŏcum
rěprěhensum qui praetěrĭtu' neglĕgentǐast.
Il y a une comédie de Diphile, les Synapothnescontes; Plaute en a fait sa pièce des
Commorientes. Dans la pièce grecque il y a au début un jeune homme qui enlève une courtisane à un leno ; c'est un passage que Plaute a laissé intouché ; or, notre auteur a emprunté ce passage pour ses Adelphes, en le rendant mot pour mot (trad. J. Marouzeau, Térence, III, CUF, p. 105).

5 Alors la préoccupation de Térence - on peut même dire sa principale préoccupation était d'éviter ce qui était arrivé à la première représentation de l'Eunuque (v. 23-34) lorsque Luscius Lanuvinus s'était mis à crier que son auteur n'était pas un poète, mais 
un voleur. La défense de Térence avait alors été malaisée : il avait pu dire seulement comme il nous le raconte dans le prologue de la pièce - qu'il avait emprunté au Colax de Ménandre, auquel tous les auteurs romains de comédies puisaient ${ }^{6}$, la figure du Flatteur et du Soldat, mais absolument pas à celui de Névius ou de Plaute, comme l'en accusait Luscius Lanuvinus, car il ne connaissait ni l'un ni l'autre :

(3) Ter., Eu. 23-34

exclámat fúrem nón poetam fábulám iamb.sen.

dedísse et nil dedísse verborúm tamén :

Colacem ésse Naevi et Plaúti véterem fábulám;

parasíti pérsonam índe ablátam et milití

si id ést peccátum, péccatum ínprudéntiást

poétae, non quo fúrtum facere stúduerít.

id ita ésse vos iam iúdicare póteritís,

Coláx Menándrist : ín ea est parasitús Coláx

et miles glóriósus : eás se nón negát

persónas transtulísse in Eúnuchúm suám

ex Graéca ; sed eas fabulás factás priús

Latínas scísse sése, id véro pérnegát.

Il s'écrie que 'c'est un voleur, non un poète qui a donné la pièce, mais qu'il n'a pas pu donner le change; qu'il existe un Flatteur, une ancienne pièce de Névius et de Plaute, que de là ont été pris les rôles du parasite et du soldat'. Si c'est là une faute, le poète a fauté par inadvertance, et sans prétendre commettre un larcin. Qu'il en soit ainsi, vous allez pouvoir en juger. Il existe de Ménandre un Flatteur, où figurent un flatteur parasite et un soldat fanfaron. L'auteur ne nie pas qu'il ait transporté ces personnages de la pièce grecque dans son Eunuque; mais qu'il ait su que ces pièces avaient été avant lui traitées en latin, voilà ce qu'il nie absolument (trad. J. Marouzeau, Térence, I, CUF, p. 224 sq.).

6 La chose était allée plus loin, au point que Térence avait repoussé la seconde représentation de l'Eunuque jusqu'à l'année $161^{7}$, en attendant, à mon avis, la mort du malivolus vetus poeta, Luscius Lanuvinus, duquel en effet il ne fait plus mention dans le prologue des Adelphes. Alors - je le répète - Térence, en présentant les Adelphes, était préoccupé et très attentif à éviter que ne se répète la situation dans laquelle il s'était trouvé lors de la présentation de l'Eunuque. Car on pouvait bien dire qu'il avait repris

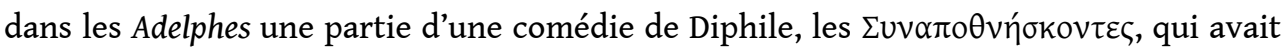
été employée par Plaute dans ses Commorientes. Pour éviter cela, Térence a établi dans le prologue des Adelphes une subtile distinction : il n'avait emprunté qu'un locus négligé par Plaute, précisément celui dans lequel Eschine enlève Bacchis au leno Sannion. Mais était-il exact que Térence s'était arrêté là, qu'il n'avait repris aucune autre partie des Commorientes de Plaute? Où recommençait Ménandre? Orazio Bianco ${ }^{8}$ affirme avec assurance : "Con la II, 2 rientriamo nel contesto menandreo degli Adelphoe », et il n'est pas le seul : Rieth ${ }^{9}$ a cherché à reconstituer Ménandre après la première scène de l'acte II, tandis que Büchner ${ }^{10}$ a écrit qu'une telle reconstitution était impossible. En effet, le matériel de Ménandre et de Diphile pouvait être mêlé jusqu'à la fin de l'acte II ${ }^{11}$ et Térence pouvait craindre que les spectateurs (et surtout ses critiques) ne pensent à un vol (furtum) des Commorientes de Plaute au-delà du locus où l'adulescens avait affaire avec le leno Sannion et où se réalisait l'enlèvement (avec paiement) de Bacchis des mains de ce dernier. Ou, plus exactement, tout au long de l'épisode durant lequel Eschine eripit meretricem au leno, on peut retrouver du matériel de Diphile. On peut dire que l'action d'eripere, " arracher, enlever avec violence ", est limitée à la première scène de l'acte II où l'esclave Parmenon donne un coup de poing au leno qui cherche à reprendre Bacchis - cela est vrai-, mais on pourrait subtiliser encore davantage en 
disant que l'enlèvement de Bacchis est déjà arrivé. Toutefois je ne veux pas le faire, car je me contente de la notice de Donat, à savoir - comme on le verra dans un instant que Térence a changé la menace de suicide qu'on trouve chez Ménandre en une menace de fuite à l'étranger. Mais quel était, sur cette question, le contenu des

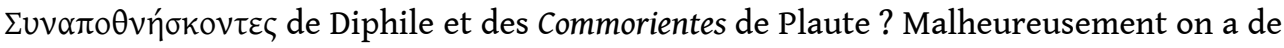
cette comédie seulement le titre. Meineke ${ }^{12}$, Webster ${ }^{13}$ et Fantham ${ }^{14}$ ont traité le sujet et s'accordent sur le fait qu'il devait s'y trouver deux jeunes qui menaçaient de se suicider probablement pour des peines de cœur, indépendamment des autres aspects du développement de la pièce. D'autre part, Donat nous dit (voir infra) que Térence a effectué des changements par rapport à Ménandre, parce que, chez celui-ci, il y avait le suicide, chez celui-là le départ pour l'étranger (e patria). Mais Donat ne nous dit rien de Diphile, dans la comédie duquel il y avait sûrement un personnage qui menaçait de mourir (de se suicider) avec un autre. Quel moyen avait Térence de prouver d'une façon incontestable qu'il ne prenait rien chez Plaute, car il avait emprunté seulement le locus des $\Sigma \cup v \alpha \pi \circ \theta v \eta ́ \sigma \kappa o v \tau \varepsilon \varsigma$, que celui-ci n'avait pas employé? Arrêtons-nous maintenant sur ce point. On y reviendra tout de suite, mais cherchons s'il y a d'autres passages dans les comédies grecques et romaines où le même personnage menaçait de faire l'une et l'autre chose comme Ctésiphon. En effet, dans la Samia de Ménandre, au vers 91, le jeune Moschion, désespéré de ne pas avoir le courage de demander à son père Déméa la permission d'épouser sa belle, Plangone (de laquelle un enfant est déjà en train de

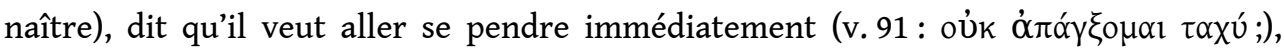
puis, aux vers 623-629, qu'il veut partir comme soldat en Asie :

(4) Men. Sam. 623-629

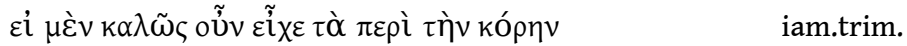

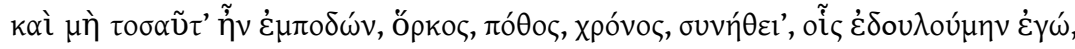

[...]

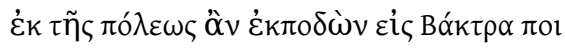

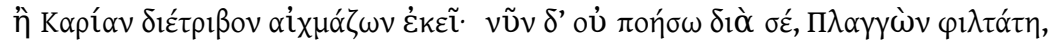

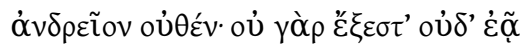

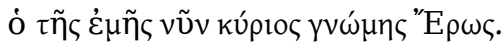

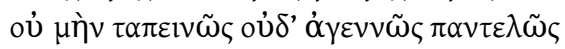

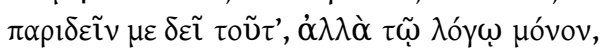

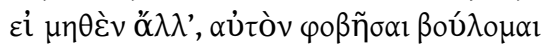

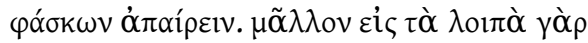

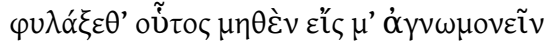

se con la ragazza avessi le carte in regola, e non ci fosse di mezzo l'amore, il giuramento, la familiarità, il tempo - tutte cose di cui sono schiavo - [...], me ne sarei andato fuori dai piedi, alla malora, in Caria oin Battriana a combattere; me lo impedisce l'amore, che è padrone della mia mente. Non devo però essere vile al punto da trascurare interamente questa cosa; se non altro, lo spaventerò almeno a parole [l'objet de cette menace est le père de Moschion], dicendo che voglio partire. Per l'avvenire sarà più attento a non farmi torto (trad. G. Paduano, Menandro, p. 319).

Mais il y a plus que cinq cents vers entre les deux références, et la première menace est seulement une exclamation : c'est bien différent de Térence, mais il est intéressant de voir l'emploi de la menace de la part de Moschion comme un moyen pour obtenir quelque chose de son père, dans ce cas un comportement plus respectueux. D'autre part il y avait aussi quelqu'un qui était vraiment allé à l'étranger comme Clinia et son père Menaedemus dans l'Heautontimorouménos (v.110-117) et qui était retourné à Athènes. 
7

Revenons alors sur les Adelphes de Térence, mais sans oublier quel était le sujet, accepté par tous, des $\Sigma u v \alpha \pi \circ \theta v \eta ́ \sigma k o v \tau \varepsilon \varsigma$ de Diphile et des Commorientes de Plaute : il y avait là deux jeunes qui voulaient mourir ensemble, peut-être pour des raisons de cœur.

8 À la fin de notre acte II qui, selon la division employée au temps de Térence, était seulement un locus, comme il l'appelle, caractérisé par l'argument traité, on voit en action Ctésiphon, Eschine, le serviteur Syrus et le leno Sannion (Bacchis est déjà entrée avec Eschine dans la maison de Micion depuis le lieu où se déroule la scène). Ctésiphon s'adresse dans un premier temps au serviteur Syrus puis à son frère Eschine, qui est sorti de la maison, et entre les deux frères s'établit un échange très intéressant :

(5) Ter., Ad. 261-275

SY.quĭd ěst? CT.quid sit? illi(u)s ŏpĕra, Syrĕ, nunc vivo. festivúm căput; iam.oct.

quin omniă sibi post putarit essě prae meo commŏdo ;

mălĕdictă famam meúm lăborem et peccatum in se tránstŭlít.

[... Eschine sort de la maison et invite son frère Ctésiphon à laisser de côté toute tristesse]

CT. ĕgo illăm [sc. tristitiam] herclě vero ŏmitto qui quĭdĕm te hăbĕam fratrem : o mi Aéschĭ

ne,

o mí germane! ah vĕrěor coram ĭn os te laudare ámplĭus,

ne ĭd adsentandi măgĕ quam quo hăběam gratum făcĕre exístŭmes. [270]

AE. ăge, ĭneptě, quăsĭ nunc nón norimu' nós inter nos, Ctésĭpho.

Hoc míhi dŏlet, nos paenĕ sero scisse et paene ĭn ĕum lŏcum

rĕdísse ut, si omnes cŭpěrent, nil tibì póssent auxĭliárǐer.

CT. pŭdébat. AE. ah stultǐťrast istaec, nón pŭdor. tam ob párvŏlam

rem páene e pătrĭa ! túrpĕ dictu. deós quaeso ŭt ǐstaec próhiběant. [275]

SY. Qu'est-ce qu'il y a ? CT. Ce qu'il y a ? C'est grâce à lui, Syrus, que je suis en vie à cette heure. Être exquis, qui a estimé que pour lui tout passait après mon intérêt ! Il a pris sur lui médisances, racontars, et ma peine et ma faute. [...] CT. Oui, par Hercule, j'y renonce, du moment que j'ai un frère tel que toi. ô mon Eschine, ô mon vrai frère. Ah j'appréhende de te louer davantage face à face, de peur que tu ne t'imagines que je le fais pour te flatter plutôt que par l'effet de ma gratitude. ES. Tais-toi, nigaud! Comme si à cette heure nous ne savions pas à quoi nous en tenir entre nous, Ctésiphon! Ce qui me peine, c'est que nous avons été prévenus presque trop tard et que nous en sommes presque venus au point que, même si tout le monde l'avait voulu, on n'aurait pu te venir en aide. CT. J'avais des scrupules. ES. Ah c'est là de la sottise, non du scrupule! Pour une si petite chose, presque s'expatrier! c'est honteux à dire. Je prie les dieux qu'ils nous préservent d'une chose pareille (trad. J. Marouzeau, Térence, III, CUF, p. 123 sq.).

On trouve alors trois références à ce que Ctésiphon, désespéré, menaçait de faire et aurait fait sans l'intervention de son frère : il s'agit de deux allusions au suicide (v. 261 et 273) ${ }^{15}$ et, à la fin (v. 275), dans la troisième, on apprend de la bouche d'Eschine que Ctésiphon avait menacé de quitter Athènes, évidemment pour devenir soldat, ainsi que l'imposait le trope. Les critiques comme Drexler et Büchner ${ }^{16}$, qui lui fait suite, ainsi que Fantham ${ }^{17}$, ont souligné que Térence a changé les données par rapport à Ménandre, car ils acceptent, avec raison à mon avis, la notice que Donat nous donne sur

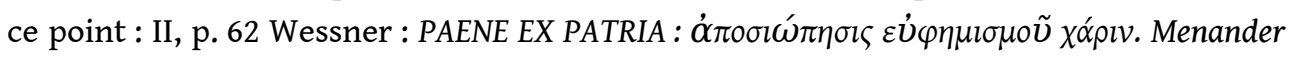
mori illum voluisse fingit, Terentius profugere. Et ils précisent, encore justement, que Térence a produit une incohérence. Mais, chez Büchner ${ }^{18}$, on lit quelque chose qui est faux d'un point de vue méthodologique, parce qu'il s'agit d'un argument $e$ silentio, mais qui s'appuie sur un fait réel : Büchner s'interroge sur les motifs de Micion, lorsqu'il se défend, dans l'acte V scène III, contre son frère Déméa qui l'accuse d'être intervenu 
aussi en faveur de Ctésiphon sans se contenter d'agir pour Eschine (v. 796 sq. : DE. [...] dictum hoc inter nos fuit, / (ex te adeo ortumst) ne tu curarés meúm/ neve égo tuom?). Pourquoi Micion ne dit pas qu'il a évité à Ctésiphon de quitter Athènes pour devenir soldat, comme il menaçait de le faire? Je ne veux pas aborder cette question, mais seulement souligner, en suivant Büchner, que Térence, après le vers 275 , ne fait plus aucune allusion à cette perspective d'exil. Il semble que Térence a employé la modification qu'il a apportée au texte de Ménandre (en remplaçant le suicide par le départ à l'étranger) pour une raison qui avait son importance à ce moment de la pièce mais n'en avait ensuite plus aucune.

9 Considérons alors cette question du suicide de Ménandre changé en exil par Térence. On a vu que le problème n'est pas de savoir où commence la scène de Diphile, mais où elle se termine complètement, où l'on peut dire avec une assurance absolue qu'elle est terminée, car je n'aurais aucune difficulté à admettre que le monologue du leno Sannion (v. 196-208) peut venir en partie de Ménandre comme l'ont supposé beaucoup de savants modernes. Il est possible que Térence ait traduit verbum de verbo la première scène où se situe la flagitatio de Sannion et ensuite entremêlé du matériel provenant de Diphile et de Ménandre. C'est une solution acceptable, si l'on donne une interprétation cohérente de la notice de Donat : que l'on trouve, aux vers 261 et 273, le suicide au lieu de l'exil va contre le fait que Térence a changé en exil le suicide de Ménandre, et on doit alors penser qu'il ne s'agit pas du suicide de Ménandre, mais de celui de Diphile. Depuis Drexler, les interprètes se sont beaucoup intéressés à cet acte II des Adelphes, mais ont laissé de côté la préoccupation de Térence, présentée dans le prologue : se distinguer de Plaute, comme il apparaît de toute évidence dans la citation $\mathrm{n}^{\circ} 2$ ci-dessus et dans une autre notice de Donat que l'on va voir plus loin.

10 L'analyse la plus fine de cette partie a été proposée par Elaine Fantham ${ }^{19}$ et je pense qu'il est juste de la retenir, car on peut en tirer des réflexions intéressantes. En effet, les interprètes modernes dont elle fait partie ont pris en considération la notice donnée par Térence lui-même (citation $n^{\circ} 2$ ) : eum hic locum sumpsit sibi / in Adelphos, verbum de verbo expressum extulit, et ils ont dit avec raison que ces lignes prouvent combien Térence a été fidèle en traduisant Diphile, mais ils ne se sont pas demandé pourquoi Térence avait émis cette affirmation. L'expression verbum de verbo a été considérée par les savants modernes comme l'élément fondamental permettant d'affirmer que le matériel de Diphile n'avait connu aucun changement. Drexler, Rieth ${ }^{20}$, Gaiser ${ }^{21}$ et Fantham ${ }^{22}$ ont cherché à reconstituer la façon dont ce locus de Ménandre se présentait à l'origine et dont il a été élaboré par Térence après l'introduction de la scène ou, plus précisément, du matériel des $\Sigma \cup v \alpha \pi \circ \theta v \eta ́ \sigma \kappa o v \tau \varepsilon \varsigma$ de Diphile, et, avec beaucoup de talent et d'intelligence critique et un peu d'imagination, ils ont proposé des reconstitutions intéressantes, mais auxquelles, pour ma part, je refuse de souscrire, tellement elles sont compliquées. Je donnerai seulement le schéma final d'Elaine Fantham (elle est d'ailleurs venue après les autres), en soulignant que tous ont mis en évidence la traduction très fidèle (verbum de verbo) de ce qui venait de Diphile, mais que tous ont travaillé avec un élément faux, c'est-à-dire la division en actes et en scènes telle qu'on la trouve aujourd'hui mais qui n'existait pas à l'époque des Adelphes de Térence et de Plaute. La démonstration de ce point sera un des résultats de cette enquête, bien qu'il s'agisse seulement de la confirmation de ce que pensait déjà Duckworth ${ }^{23}$, avec toutefois une précision importante. Il faut donc envisager ce que pouvait être le texte de Diphile pour avoir une idée du matériel dont a pu hériter le locus cité par Térence, c'est-à-dire 


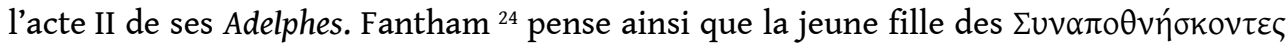
n'était pas esclave mais libre, et qu'allait se produire dans cette comédie une anagnorisis, annoncée dans un prologue, comme le prouveraient les vers 193 sq.: $A E$. Neque vendundam censeo / quae liberast; nam ego liberali illam adsero causá manú. Pour respecter le texte de Diphile qu'il traduisait verbum de verbo, Térence aurait changé seulement la référence à la jeune fille dans son prologue, où elle est qualifiée de meretricem. Un point de détail est que le leno Sannion dit (encore une fois dans la scène de Diphile) qu'il n'a aucune affaire avec Eschine: cela est vrai dans les Adelphes de Ménandre où l'amant de Bacchis est Ctésiphon, et non Eschine, comme dans les $\Sigma \cup v \alpha \pi \circ \theta v \eta ́ \sigma \kappa o v \tau \varepsilon \zeta$ où c'est un ami (chez Ménandre un frère) qui agit pour un autre. Et ceci est l'hypothèse la plus plausible « and lends some support to the theory that the synapothnescontes dealt with a plot based on a threatened lovers' suicide pact, which has led the abduction of the girl by the "Aeschinus" character on his friend's behalf ${ }^{25}$ ”. Diphile, si l'on considère aussi le Rudens de Plaute dans les parties qui viennent de son œuvre, est bien plus dur et grossier que Ménandre, il met en scène un esclave qui donne des coups de poing à un leno exactement comme dans les Adelphes de Térence (Plaut., Rud. 711) 26. Gaiser pensait que, dans la pièce de Ménandre, la rencontre d'Eschine et de Ctésiphon venait avant la scène où Eschine et Syrus affrontent le leno qui les a rejoints, et Elaine Fantham suppose également que l'acte II s'ouvrait avec l'arrivée d'Eschine à la maison de Micion après que celui-ci l'a quittée pour aller au forum. Dans un prologue précédent, chez Ménandre, les auditeurs avaient appris qu'Eschine était intervenu pour sauver son frère, et l'adulescens avait ainsi gagné la sympathie des spectateurs. "Act II begins with his arrival [l'arrivée d'Eschine à la maison de Micion]; a monologue (or perhaps a speech addressed to the silent girl). He goes into the house in search of Ctesipho or Syrus; finds Syrus, who has returned ahead of Ctesipho; meanwhile Ctesipho enters with a soliloquy; Syrus, emerging from the house to look for him, greets him, calling him back to earth. After this the action proceeds along the lines that I indicated in part I, with the pimp's entrance delayed until after the brothers' reunion; his private negotiations with Syrus lead to a brief, final scene in which Aeschinus is followed by Sannio and Syrus to the market place to complete the sale, and the act ends with the satisfaction of all parties ${ }^{27}$ ». D'autre part, Fantham ${ }^{28}$ pense que le point où Térence reprend Ménandre, en laissant Diphile, se trouve aux vers 200-201, dans le monologue où le leno Sannion, après s'être lamenté sur tout ce qu'il a subi d'Eschine, envisage la possibilité de traiter avec le jeune homme pour sauver son argent, le prix de la belle. C'est possible, mais on n'a aucune preuve, le leno pouvait aussi bien se disposer aux mêmes pourparlers chez Diphile.

11 En étudiant encore mieux Térence, je dirais que les interprètes modernes n'ont pas suffisamment évalué ce qu'il voulait signifier par verbum de verbo expressum extulit (on doit citer tous les mots qu'il emploie). Je ne pense pas qu'il voulait simplement dire qu'il avait fait une traduction fidèle - pourquoi le souligner ?-, mais plutôt préciser qu'il avait fait une traduction fidèle sans rien ajouter au locus où l'adulescens [...] lenoni eripit/ meretricem, un lieu que Plaute n'avait pas touché (eum Plautus locum/ reliquit integrum), et qu'il n'y avait rien introduit qui pouvait venir des Commorientes. C'était donc un lieu de Diphile qu'il présentait aux spectateurs romains après l'avoir isolé de son contexte (expressum extulit) mais sans rien changer de son contenu verbal. Ceci est, à mon avis, le sens de verbum de verbo expressum extulit, parce qu'il n'y avait aucune raison pour Térence de dire qu'il avait fait une traduction fidèle : fidèle signifiait, à mon avis, qu'il n'y avait pas ajouté d'expressions tirées des autres parties des 
$\Sigma \cup v \alpha \pi \circ \theta v \eta ́ \sigma \kappa o v \tau \varepsilon \varsigma$, parties qui pouvaient se trouver dans les Commorientes de Plaute et n'étaient plus, pour cette raison, integrae. Cette explication est la même que celle que nous a donnée Donat à propos de verbum de verbo expressum (II, 11 Wessner) : VERBUM DE VERBO EXPRESSVM EXTVLIT hic approbatur uere de Graeco esse sublatum, non de Plauto, ut dixit ${ }^{29}$ aduersarius. Et prenons garde au fait qu'il ne s'agit pas seulement d'une confirmation de mon interprétation de ces mots, mais aussi du souci majeur de Térence d'éviter l'accusation d'avoir puisé chez Plaute; il s'agit donc d'une confirmation de toute ma thèse concernant la volonté de Térence de prouver qu'il n'avait pris rien de Plaute, même si je ne peux pas dire que le raisonnement de Donat fut le même que celui que nous avons suivi ci-dessus.

Mais, s'il est vrai que le souci de Térence était de démontrer que, dans ce locus, il n'avait absolument rien puisé chez Plaute, se posait pour lui, comme pour nous, la question de savoir où se terminait de façon indiscutable le locus en question. Ici on ne doit pas seulement considérer ce que Térence avait fait, mais aussi ce que les spectateurs, et surtout les critiques comme Luscius Lanuvinus, pouvaient penser, ou mieux, ce que Térence craignait que les critiques ${ }^{30}$ et les spectateurs ne pensent, car telle était justement la préoccupation de Térence. Pour ma part, je croirais volontiers que la partie tirée de Diphile ne va pas beaucoup au-delà de ce qui est pour nous la première scène de l'acte II, mais le locus qui concernait le rapport Eschine $\sim$ Sannion à l'égard de la jeune Bacchis arrive à la fin de ce que nous considérons comme l'ensemble du deuxième acte. Je le répète : la crainte de Térence touchait à ce qui pouvait éveiller les soupçons des critiques et des spectateurs, et ils pouvaient naître jusqu'à la fin de l'acte, c'est-à-dire du locus où Eschine enlève la belle au leno en la payant. Il est vrai qu'Elaine Fantham a pensé que, dans la pièce de Diphile, la jeune femme était libre comme tendrait à le prouver le vers 195, nam ego liberali illam adsero causa manu, et cela serait confirmé par une anagnorisis. Mais, encore une fois, si l'on considère toute l'expression, on en conclut plutôt qu'Eschine voulait dire autre chose : il était prêt à en faire une manumissio, car il avait la jeune fille entre les mains, mais il était prêt aussi à payer pour elle le prix que Sannion avait payé, viginti minae, v. 191 : AE. Minis viginti tu illam emisti (quae res tibi vortat male!): / argenti tantum dabitur, et il laisse au leno l'alternative, v. 193-195: AE. Neque vendundam censeo / quae liberast; nam ego liberali illam adsero causá manú. / nunc vide utrum vis, argentum accipere an causam meditarí tuam. / delibera hoc dum ego redeo, leno. Ceci revient à dire: "Toi, Sannion, tu as deux possibilités : prendre l'argent en nous vendant Bacchis ou démontrer contre moi au tribunal que Bacchis est ton esclave et non pas la mienne, et, pour démontrer qu'elle est la mienne, je la déclare libre avec cet affranchissement $»$. Il ne me semble pas qu'Eschine soupçonne que Bacchis était libre et citoyenne attique (d'où l'anagnorisis, etc.). On pourrait penser soit que Térence a opéré quelques changements (mais c'est impossible, parce qu'il a traduit verbum de verbo), soit qu'Eschine présente de façon floue un élément qui sera précisé plus loin, mais ici Bacchis est une esclave et seul un jeu de l'imagination peut la transformer en citoyenne attique dans l'attente d'être reconnue ${ }^{31}$.

Alors, pour les spectateurs romains qui ne connaissaient pas la suite des $\Sigma \cup v \alpha \pi \circ \theta v \eta ́ \sigma \kappa o v \tau \varepsilon \varsigma$ comme la connaissent les savants modernes (avec l'aide de l'imagination), Bacchis était erepta des mains du leno, sans qu'il y ait vraiment d'affaire à traiter au tribunal ni quoi que ce soit d'autre, seulement à la fin du locus, c'est-à-dire à la fin de l'acte II. 

(conformément à Diphile et à Ménandre) et exil (changement de Térence, comme nous le dit Donat). La raison de cette modification (inutile du point de vue de la structure de la pièce) est évidente, c'était encore une fois le besoin de montrer de manière irréfutable aux critiques et spectateurs romains que lui, Térence, n'avait rien pris des Commorientes de Plaute, qu'il s'arrêtait avant d'entrer sur le terrain des Commorientes parce qu'il n'y avait aucune menace de mort: Ctésiphon voulait aller $e$ patria, il ne voulait pas se suicider comme les personnages des Commorientes. On n'avait donc pas affaire avec les Commorientes de Plaute, parce qu'il n'y avait aucun moriens. Avec trois mots, paene e patria, Térence a éliminé tout soupçon de furtum des Commorientes de Plaute. À partir de là, toute accusation de ce genre était impossible, même en laissant

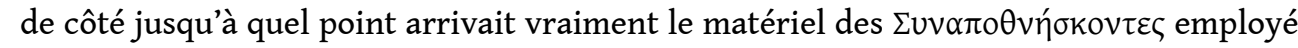
par Térence, même si je pense - opinion tout à fait personnelle - qu'il arrivait lui aussi jusqu'à ce point.

15 Ainsi nous avons trouvé une réponse à la question de savoir pourquoi Térence a effectué un changement par rapport à Ménandre, mais il y a une autre question, liée à la première : pourquoi Térence n'a-t-il pas changé les trois menaces de Ctésiphon et a attendu à la fin (v. 275), lorsque le leno était d'accord pour vendre Bacchis à Eschine (v. 276: AE. Quid ait tandem nobis Sannio? SY.iam mitis est). On doit aussi donner une réponse à cette question pour compléter notre démonstration et pour comprendre la technique de Térence. On a vu qu'il avait peur que les critiques ne pensent qu'il avait volé Plaute et ses Commorientes. Térence avait dit dans le prologue que, dans le locus

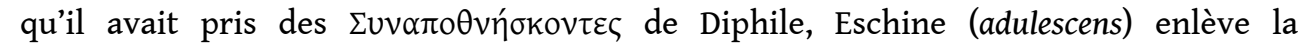
courtisane au leno (lenoni eripit / meretricem). L'enlèvement a eu lieu avec usage de la violence et versement d'une somme d'argent, l'un n'excluant pas l'autre, Eschine le dit clairement (v. 191). Rentrons dans l'optique de Térence : il portait une grande attention à ce que les critiques et les spectateurs soupçonneraient, et qu'est-ce qu'un critique romain pouvait soupçonner? Il y avait deux possibilités : il pouvait croire que la dette vis-à-vis de Diphile se limitait à la violence ou qu'il y avait déjà chez lui le paiement.

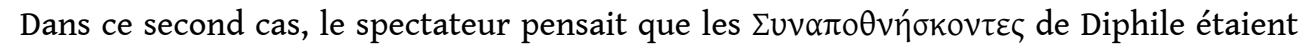
encore présents jusqu'au paiement. Mais nous savons que, dans cette comédie de Diphile, deux personnages menaçaient de se suicider. Si Térence avait changé la première et la deuxième menace de Ctésiphon, en passant du suicide à l'exil, il serait apparu comme un menteur aux yeux de ces spectateurs qui pensaient que l'enlèvement de la courtisane et le locus de Diphile se complétaient par l'accord donné au paiement de vingt mines au leno Sannion, car Térence avait dit qu'il avait traduit Diphile verbum de verbo expressum et, chez Diphile, il y avait menace de suicide, non d'exil. Le seul endroit où Térence devait montrer que le locus de Diphile se terminait (et ainsi le fantôme des Commorientes disparaîtrait complètement) ne pouvait alors être que celui où il a placé l'exil au lieu du suicide, au vers 275 , cette porte qui laissait en dehors les Commorientes de Plaute, à la fin du lieu où était conclu l'enlèvement de Bacchis de chaque point de vue.

Quelles sont les conséquences de cette solution? La première est que Térence n'avait pas utilisé de division en actes et en scènes, car il aurait pu alors désigner l'un ou l'autre comme le terme de l'utilisation du matériel de Diphile, et ceci dans la dernière de ses pièces: il s'agissait d'une technique qui n'avait pas encore été suffisamment développée. L'autre conséquence est celle de laquelle nous sommes partis : le mensonge 
d'Eschine. Il fait partie de l'échec subi par Micion à la fin de la pièce : l'éducation de Déméa a échoué avec Ctésiphon, mais Micion aussi échoue, car il doit donner un fonds à Hegion, libérer Syrus et sa femme, et se marier avec la vieille Sostrata. Il cherche à résister à cette dernière sollicitation de son frère Déméa, mais Eschine appuie cette demande en disant, ce qui est un mensonge, qu'il l'avait promis à Sostrata et Pamphila, chose impossible, parce qu'il n'est pas rentré dans la maison de Sostrata après avoir reçu de son père adoptif Micion la permission d'épouser Pamphila. Qu'il s'agit d'un mensonge, c'est là l'opinion de Drexler, Rieth ${ }^{32}$, Büchner ${ }^{33}$ et Fantham ${ }^{34}$, tandis que pour Pöschl ${ }^{35}$ il n'y a en réalité aucun échec de Micion. Cette question a déjà été traitée par Lessing et on a discuté pour savoir si l'échec de Micion était plus fort chez Ménandre ou chez Térence. En effet, il semble que pour de nombreuses raisons l'échec est plus fort chez Térence selon ce que disait déjà Donat, au vers 938 (II, p. 176 Wessner) : EGO NOVVS MARITUS apud Menandrum senex [sc. Micio] de nuptiis non gravatur, c'est-à-dire "il accepte de bon gré les noces avec la vieille Sostrata », Hégion était le frère de Sostrata, l'ager que lui donnait Micion restait donc dans le үévoc. Mais chez Térence il y a le mensonge d'Eschine. Se trouvait il aussi chez Ménandre ? Pour ma part, je dirais à première vue que oui, et que chez Ménandre également l'éducation de Micion péchait par excès d'indulgence envers Eschine: après avoir prouvé que l'éducation rigide de Déméa a abouti à de mauvais résultats avec Ctésiphon, Ménandre démontrerait que l'éducation trop indulgente de Micion est également manquée, parce que Ménandre était influencé par la doctrine du Portique, qu'il avait apprise de Théophraste, et pensait qu'il y avait la medietas. C'est la direction que prennent Johnson ${ }^{36}$ et Lord ${ }^{37}$, tandis que Gaiser ${ }^{38}$ nous a montré de quelle importance avait été pour Ménandre la doctrine aristotélicienne. D’autre part, Aristote, Po. 1449 a 32-37,

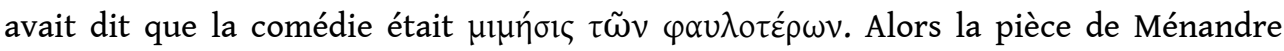
n'était pas seulement un spectacle, mais un moyen pour mettre en évidence les faiblesses et les vices des hommes, et je pense que Térence a déjà trouvé chez Ménandre l'échec de Micion, et qu'il l'a exagéré pour des raisons techniques, parce qu'il a éliminé certaines de ses références. Et le mensonge? Mais s'agit-il vraiment d'un mensonge ? Pourquoi est-on obligé de penser à un mensonge ? Parce que, répond Rieth ${ }^{39}$, Eschine ne pouvait pas promettre de demander à Micion d'épouser Sostrata avant d'avoir obtenu lui-même de celui-ci la permission d'épouser Pamphila. Mais je me demande s'il n'était pas possible qu'Eschine ait tenu ce discours à Sostrata : «Si mon père Micion me donne la permission d'épouser Pamphila, je promets que je lui demanderai qu'il t'épouse toi-même, Sostrata ». Alors nous ne sommes pas obligés de penser qu'Eschine a dit un mensonge. Au contraire, il est sûr qu'il a péché d'un autre point de vue : aux vers 710 sq., il avait affirmé qu'il n'aurait jamais rien fait que Micion réprouve (Ter., Ad. $710 \mathrm{sq}$.: itaque adeo magnam mi iniicit sua commoditate curam, / ne inprudens forte faciam quod nolit: sciens cavebo, «et voilà qu'ainsi par sa gentillesse il m'inspire un vif souci de ne pas faire inconsidérément des choses qu'il ne voudrait pas ; je me garderai à bon escient $\left.{ }^{40} »\right)$. Et, sans aucune cohérence, il intervient pour que Micion épouse Sostrata contre la volonté de Micion. Le vice d'Eschine est donc le manque de cohérence. C'est la même incohérence qu'on trouve aux vers 272-275, où Eschine change le suicide de Ctésiphon en exil. Il s'agissait là d'une intervention de Térence, comme nous le dit Donat, puis, au vers 942, Donat ajoute apud Menandrum senex de nuptis non gravatur. Térence est intervenu dans les deux cas. La construction de Térence est parfaite et, aux vers 272-275, il n'a pas seulement opéré un changement par rapport à Ménandre pour prouver qu'il n'avait rien à voir avec les Commorientes de 
Plaute, mais il est resté fidele au caractère d'Eschine, qui était quelqu'un d'impulsif et d'incohérent.

17 Je dois aborder un autre point qui a à voir avec Plaute. Comme on l'a vu dans le prologue de l'Eunuque, Térence se défend en disant (v. 27) : si id est peccatum, peccatum imprudentiast / poetae (citation $\mathrm{n}^{\circ} 3$ ). Il ne connaissait pas Névius ni Plaute. Mais dans le prologue des Adelphes, il semble très bien connaître Plaute. Il l'a lu, peut-être avec l'aide du grand acteur Ambivius Turpion ${ }^{41}$. Il a pris un vers du Trinummus de Plaute, comme cela a été souligné par Bianco ${ }^{42}$ et par Klose ${ }^{43}$, en le modifiant un peu : Ter., Ad. 22 sq. : de(h)inc ne exspectetis argumentum fabulae / senés qui primi venient î partem aperient Plaut., Trin. 16 sq. : sed de argumento ne exspectetis fabulae : senes qui huc venient, i rem vobis aperient (en gras les mots communs). Le modèle du Trinunnus était Philemon (Plaut., Trin. 19). Lentano ${ }^{44}$, à son tour, a montré que Térence a élaboré le monologue initial de Micion (v. 35-38) en empruntant à Plaute, Mil. 718-722 : PE. Pol si habuissem, sati' cepissem miseriarum e liberis : / continuo excruciarer animi : si ei fort' fuisset febris, / censerem emori ; cecidissetve ebrius aut de equo uspiam, / metuerem ne ibi diffregisset crura aut cervices sibi. Ter., Ad. 35-38 : ego quia non rediit filius quae cogito et / quibu' nunc sollicitor rebu'! ne aut ille alserit / aut uspiam ceciderit aut praefregerit / aliquid ${ }^{45}$. Pour ma part, j'ai vu dans la conclusion de l'Eunuque une parodie de l'elegantia du soldat Pirgopolinices du Miles de Plaute: Ter., Eu. 1093, GN. Dixin ego in hoc [dans le soldat de l'Eunuque de Térence, Thrason ; celui qui parle est le parasite Gnathon] esse vobis Atticam elegantiam ? Térence ne craignait donc pas d'employer des expressions de Plaute, des mots de Plaute, mais il avait peur qu'on puisse l'accuser de furtum dans la grande question de la contaminatio et $\mathrm{du}$ furtum. La contamination réduisait les pièces grecques que les poètes romains pouvaient employer sans risquer de commettre de furtum. C'était une question technique dans laquelle il y avait une sorte de 'copyright' sur les pièces grecques traduites par les poètes romains. Il n'y avait pas de 'copyright' sur les mots latins. La seule manière d'éviter cela était de faire comme Térence, de traduire une scène, ou plutôt un locus, en veillant bien à ce qu'il s'agisse d'un locus qui n'avait pas encore été repris par un poète romain et en indiquant très précisément où commençait et où se terminait cet emploi. Térence avait bien compris la leçon de l'Eunuque, avait lu Plaute (comme le prouvent les références que l'on vient de voir) et avait fixé les termes précis de ce qu'il avait pris de Diphile.

18 Et Donat? Quel a été l'apport de Donat à la solution de ce problème, celui de savoir pourquoi Térence avait changé avec trois mots, paene e patria, le suicide de Ménandre en exil, si toutefois ma solution est juste? Je dois dire que l'apport de Donat a été énorme, trois éléments le prouvent : $1^{\circ}$ il y a eu un changement à la fin de l'acte II $; 2^{\circ} \mathrm{ce}$ petit changement ne pouvait pas passer inaperçu, ainsi a-t-il été observé par Donat, entre autres parce qu'il s'agissait d'un changement trop immédiat dans la bouche de l'incohérent Eschine ; $3^{\circ}$ les deux références à la menace de suicide qu'on trouve chez Térence étaient du matériel issu de Diphile que Térence n'avait pas modifié parce qu'il était fidèle à verbum e verbo expressum extulit ${ }^{46}$. S'il y avait eu du matériel de Ménandre, Térence l'aurait changé comme il a changé la troisième référence, si l'on tient pour exacts les mots de Donat. On peut penser qu'il s'est comporté d'une façon stupide c'est une accusation qu'on trouve, par exemple, pour la fin de la pièce où on dit que Térence a ruiné la conclusion de Ménandre. Mais dans ce cas c'est plutôt le contrepoint suicide exil qui met en relief le changement et c'était ce que Térence voulait souligner de toute évidence, à savoir qu'à partir de ce point-là il n'y avait aucun suicide et aucune 
référence possible aux Commorientes de Plaute. Il avait fermé la porte à toute influence, directe ou indirecte, des Commorientes de Plaute. J'ai douté à peu près dix ans avant d'arriver à la solution que j'ai trouvée et, sans Donat, j'aurais navigué moi-même sur la mer de l'imagination.

Il y a eu alors trois niveaux d'interprétation, du point de vue littéraire : le premier, substantiel, de Donat; le deuxième qui nous a porté à trouver des aspects liés au rapport de Térence avec les autres auteurs comiques romains, Luscius Lanuvinus, Plaute; et le troisième niveau où l'on peut voir une confirmation du caractère éthique et éducatif de la comédie de Ménandre qui incitait à réfléchir sur la faiblesse humaine. Une pièce riche que cette comédie représentée aux Jeux funèbres de Paul-Émile et dans les ludi donnés par Q.Fabius Maximus ${ }^{47}$, le fils aîné de Paul-Émile : on y trouvait de la violence (coups de poing comme dans le Rudens de Plaute) ${ }^{48}$ mais aussi la réflexion philosophique des hellénistes romains, tels que les Scipions. Et elle s'adaptait bien à P. Cornelius Scipio Aemilianus, le plus jeune fils de Paul-Émile, celui qui donnait comme spectacle les deux comédies, les Adelphes et l'Hécyre de Térence. Mais sans le grammairien Donat et ses trois interventions, hic approbatur uere de Graeco esse sublatum, non de Plauto, ut dixit aduersarius (v. 11), Menander mori illum uoluisse fingit, Terentius profugere (v. 275) et le célèbre apud Menandrum senex de nuptiis non grauatur (v. 942), on n'aurait pas eu le niveau de base duquel sont issus les autres.

Et Térence? Térence était un jeune homme plein d'espoir et de crainte comme beaucoup de jeunes, plein de génie comme peu d'hommes.

\section{BIBLIOGRAPHIE}

BIANCO O. 1962. Terenzio: problemi e aspetti dell'originalità, Nuovi saggi 41, Rome.

BÜCHNER K. 1974, Das Theater des Terenz, Bibliothek der klassischen Altertumswissenschaften.

1. Reihe 4, Heidelberg.

BUREAU B. 2011, « Térence moralisé : les sententiae de Térence selon le commentaire attribué à

Donat ", in C. Mauduit, P. Paré-Rey (éds), Les maximes théâtrales en Grèce et à Rome : transferts, réécritures, remplois, Actes du colloque organisé les 11-13 juin 2009 par l'université Jean-Moulin Lyon 3 et

l'ENS de Lyon, Coll. du Centre d'études romaines et gallo-romaines 37, Paris, p. 157-175.

CALBOLI G. 1991, «Il miles gloriosus di Terenzio e l'infinito storico latino », in Studi di filologia classica in onore di Giusto Monaco, Palerme, p. 599-632.

CALBOLI G. 1996, « Maecius Tarpa », in Orazio: enciclopedia oraziana. I, L'opera, I luoghi, Le persone, Rome, p. 803-805.

CALBOLI G. 1999, « Rhétorique et droit romain », REL 76, p. 158-176.

CALboli G. 2002, « Les Adelphes de Térence, la Fortune et le Dialogue », Veleia 18-19, p. 357-371.

CAROSI G. 2006, Cecilio Stazio e il PHerc.78: Obolostates sive Faenerator [Dottorato di Ricerca in Filologia Greca e Latina, Univ. di Bologna]. 
CERESA-GASTALDo A. (éd.) 1988, Gerolamo. Gli uomini illustri, Biblioteca Patristica 12, Florence. DUCKWORTH G.E. 1994, The Nature of Roman Comedy: A Study in Popular Entertainment, $2^{\mathrm{e}}$ éd., Londres (Princeton, 1952 $2^{1}$.

FANTHAM E. 1968, « Terence, Diphilus and Menander, A re-examination of Terence, Adelphoe, Act II ", Philologus 112, p. 196-216.

FANTHAM E. 1971, « Heautontimorumenos and Adelphoe: a Study of Fatherhood in Terence and Menander », Latomus 30, p. 970-998.

FORTENBAUGH W.W., SCHÜTRUMPF E. (éds) 2000, Demetrius of Phalerum: Text, Translation and Discussion, Rutgers University Studies in Classical Humanities 9, New Brunswick - Londres.

GAISER K. 1967, « Menander und der Peripatos », A\&A 13, p. 8-40.

GARTON C. 1972, Personal Aspects of the Roman Theatre, Toronto.

GRATWICK A.S. (éd.) 1987, Terence. The Brothers, Classical Texts, Warminster.

GotTsChalK H.B. 1999, « Demetrius of Phalerum: A Politician among Philosophers and a Philosopher among Politicians ", in W.W. Fortenbaugh, E. Schütrumpf (éds), Demetrius of Phalerum: Text, Translation and Discussion, Rutgers University Studies in Classical Humanities 9, New Brunswick - Londres, p. 367-380.

GUARDì T. (éd.) 1974, Cecilio Stazio. I frammenti, Hermes. Collana di testi antichi 9, Palerme. JOHNSON W.R. 1968, « Micio and the Perils of Perfection », California Studies in Classical Antiquity 1, p. 171-186.

KLOSE D. 1966, Die Didaskalien und Prologe des Terenz [Thèse : Lettres : Freiburg i. Br.].

KLOWSKI J. 2000, « Terenz' Adelphen und die modernen Erziehungsstile », Gymnasium 107, p. 109-127.

LEFÈVRE E. 1969, Die Expositionstechnik in den Kömodien des Terenz, Darmstadt.

LENTANO M. 1996, «I dispiaceri di un padre. Fra Menandro e Terenzio », BStudLat 26, p. 3-8.

LORD C. 1977, « Aristotle, Menander and the “Adelphoe” of Terence », TAPhA 107, p. 183-202.

MARTIN R.H. (éd.) 1976, Terence. Adelphoe, Cambridge Greek and Latin Classics, Cambridge.

MASSIONI M. 1998, Il TРОПОГ e Terenzio: Teofrasto e Menandro, Papers on Rhetoric, Bologne.

MEINEKE A. 1839, Historia critica comicorum Graecorum, Fragmenta comicorum Graecorum I, Berlin. PADUANO G. 1980, Menandro. Commedie, Biblioteca 6, Milan.

PÖschl V. 1975, Das Problem der „Adelphem“ des Terenz, Sitzungsberichte der Heidelberger Akademie der Wissenschaften. Phil.-hist. Klasse 4, Heidelberg.

RIETH O. 1964, Die Kunst Menanders in den „Adelphem“ des Terenz, mit einem Nachwort hrsg. von K. Gaiser, Hildesheim.

SCULLARD H.H. 1960, « Scipio Aemilianus and Roman Politics », JRS 50, p. 59-74.

SPRANGER P.P. 1960, Historische Untersuchungen zu den Sklavenfiguren des Plautus und Terenz, Akademie der Wissenschaften und der Literatur in Mainz. Abhandlungen der geistes- und sozialwissenschaftlichen Klasse 8, Wiesbaden. 
STORK P., VAN OPHUIJSEN J.M., DORANDI T. 1999, « Demetrius of Phalerum: The Sources, Text and Translation», in W.W. Fortenbaugh, E. Schütrumpf (éds), Demetrius of Phalerum: Text, Translation and Discussion, Rutgers University Studies in Classical Humanities 9, New Brunswick - Londres, p. $1-310$.

TRACY S.V. 1999, « Demetrius of Phalerum: Who was He and Who was He Not? », in W.W. Fortenbaugh, E. Schütrumpf (éds), Demetrius of Phalerum: Text, Translation and Discussion, Rutgers University Studies in Classical Humanities 9, New Brunswick - Londres, p. 331-345.

TROMARAS L., PETERSEN M. (éds.) 1994, P. Terentius Afer Eunuchus: Einführung, kritischer Text und Kommentar, Hildesheim.

WALBANK F.W. 1979, A Historical Commentary on Polybius. III, Commentary on Books XIX-XL, Oxford.

WEBSTER T.B.L. 1960, Studies in Menander, $2^{\mathrm{e}}$ éd., Publications of the University of Manchester 309, Classical Series 7, Manchester (19501).

Voir la table d'Albrecht Dürer, Roemer Tafel 22, Albrecht Dürer, 1471 bis 1528: das gesamte graphische Werk. II, Druckgraphik, Einleitung von Wolfgang Hütt, Berlin, Henschelverlag, 1968, S. 1226, und besonders mein 'Bozzetto'.

\section{NOTES}

1. Lorsqu'on parle de Donat et de son commentaire de Térence, on doit entendre non seulement Donat, mais aussi «la tradition interprétative térentienne telle qu'elle existait au milieu du IV siècle ", comme cela a été mis en évidence par Bureau 2011, p. 157 sq., sans exclure l'existence d'un commentaire de Donat en faveur d'un objet qu'on ne connaît pas. Pour ce qui concerne la vie de saint Jérôme, voir Ceresa-Gastaldo 1988, p. 9-18.

2. Rieth 1964.

3. Büchner 1974.

4. Ter., Hau. 93-117 (Clinia a quitté Athènes pour aller en Asie servir de soldat du roi) et 175-183 (retour de Clinia d'Asie). Ainsi avait fait son père Ménédème, lui aussi rentré dans sa ville, Athènes.

5. La division en actes date de l'époque de Varron ou de Varron lui-même, elle n'existait pas du temps de Térence, comme on va le démontrer dans cet article d'une façon que je dirais incontestable. Cependant, la première partie des A $\delta \varepsilon \lambda \varphi o$ de Ménandre se termine par un monologue de Micion (v. 141-154), qui signale très clairement qu'il s'agit d'une conclusion. Mais, à la fin de notre étude, on pourra tirer des conclusions intéressantes.

6. On pouvait emprunter aux Grecs, parce que - je suppose - les Grecs étaient des deditici, c'està-dire qu'ils se trouvaient en situation d'esclavage par rapport aux Romains, et surtout parce que leurs œuvres étaient déjà des communia, comme je l'ai soutenu dans Calboli 1999, p. 158-176, tandis que les Romains évitaient de puiser chez d'autres Romains, ainsi du temps d'Ennius qui s'était abstenu de décrire la première guerre punique déjà représentée par Naevius (voir Cic., Brut. 75 sq.).

7. La chronologie de l'Eunuque est bien compliquée, parce que même la tradition manuscrite et la notice de la Vita Terenti fournissent des données contradictoires (voir Marouzeau, Notice, p. 215). Il semble que l'Eunuque a été présenté comme la deuxième des comédies de Térence (ainsi dans les manuscrits, mais Donat dit : edita tertium est, et, dans la Vita Terenti 3, p. 5 Wessner, il nous précise que l'Eunuque a été représenté deux fois le même jour, bis die acta est), la pièce a été donnée après l'Andria (166), si l'on en croit la didascalie (facta secunda), et la représentation a eu lieu en 161 (M.Valerio C.Fannio coss.), voir aussi Klose 1966, p. 19 ; Calboli 2002, p. 363 sq. Mais il est 
possible que Térence se taise sur Luscius Lanuvinus dans le prologue des Adelphes, car il avait dit dans celui du Phormion, $22 \mathrm{sq}$. : de illo iam finem faciam dicundi mihi, / peccandi quom ipse de se finem non facit, si ces mots ne se référent pas seulement à ce que Térence est en train de faire dans le Phormion. Comparer à Klose 1966, p. 140 sq.

8. Bianco 1962, p. 184.

9. Rieth 1964, p. 52-59.

10. Büchner 1974, p. 383.

11. Voir la discussion et l'indication de tous ceux qui ont fait des propositions à cet égard dans Büchner 1974, p. 382.

12. Meineke 1839, p. 456.

13. Webster 1960.

14. Fantham 1968, p. 199, n. 1.

15. Dans la deuxième référence, Eschine dit que «nous en sommes presque venus au point que [...] on n'aurait pu te venir en aide" et cela pouvait signifier deux choses: que le leno Sannion avait déjà pris la mer avec Bacchis ou que Ctésiphon s'était déjà suicidé. Mais la première éventualité était-elle vraiment sans remède? Dans le Miles de Plaute, il y a un déplacement d'Athènes à Éphèse et un autre déplacement de Cyrène en Sicile avait été amorcé dans le Rudens. Il s'avère ainsi qu'Eschine ne disait vrai que dans le cas du suicide.

16. Büchner 1974, p. 377.

17. Fantham 1968.

18. Büchner 1974, p. 411 et 413.

19. Fantham 1968.

20. Rieth 1964.

21. Gaiser 1967.

22. Fantham 1968.

23. Duckworth 1994, p. 98-101. Duckworth conclut ainsi sa discussion sur cette question p. 101 : « In summary, we may conclude that neither Plautus nor Terence applied any rule of act-division to their comedies, and that the plays were usually on the stage with complete continuity of action $"$.

24. Fantham 1968, p. 202.

25. Fantham 1968, p. 203.

26. Garton 1972, p. 77, met en évidence que Diphile a eu beaucoup de succès à Rome : "the success of his plays with Roman audiences are attested by Plautus' use of his work for the Rudens, Casina, Commorientes, and perhaps the Vidularia, and the fact that Terence was later to draw upon Diphilos for a scene of the Adelphoe. Other things being equal, Luscius might very well choose to adapt Diphilos' Thesauros». Pourquoi Térence a-t-il choisi lui aussi Diphile est une question intéressante. Peut-être voulait-il suivre une tendance plautinienne (sans voler Plaute ou apparaître comme un voleur de Plaute). Un rapport avec Plaute a été justement vu par Lentano 1996 dans le monologue de Micion à la fin du premier acte. On verra plus loin ce point.

27. Fantham 1968, p. 214-215.

28. Fantham 1968, p. 204.

29. Je lis dixit avec le manuscrit Oxon.Bodleian.Canon.lat. 95, tandis que les autres manuscrits et Wessner lisent dicit, et je le fais pour laisser ouverte la possibilité que Donat se réfère à ce que l'adversaire (aduersarius), Luscius Lanuvinus, avait fait effectivement à la représentation de l' Eunuque. Car dicit renvoie à l'intervention d'un adversaire (à Luscius Lanuvinus, qui n'est pas mentionné dans les Adelphes et qui était, peut-être, déjà mort?), une intervention qui, en tout cas, n'a pas lieu dans les Adelphes. Il va sans dire que dixit s'accorde avec mon explication bien mieux que dicit.

30. Dans le groupe de ces critiques pouvaient se trouver des membres du collegium scribarum histrionumque qui, comme le Maecius Tarpa d'Horace, pouvaient être juges des poètes (Hor., S. 1, 
10, 37-39 : iudice Tarpa ; Ars 386-388 : in Maeci descendat iudicis auris ; Porph., Ad S. 1, 10, 38 : in aede Musarum ubi poeta<e> carmina sua recitabant; Cic., Fam. 7, 1, 1 ; voir Calboli 1996, p. 803-805, s.v. Maecius Tarpa).

31. Gratwick 1997, p. 238 : «the generally favoured explanation is that the young man in Diphilus must have been bluffing, and that that is also what Terence means us to understand; Aeschinus is threatening to frame Sannio with false evidence ». Voir aussi Martin 1976, p. 133, qui pense à un 'bluff' d'Eschine. Pour ma part, j'estime qu'Eschine fait plus qu'un 'bluff', il simule et accomplit aussi un affranchissement en laissant au leno la charge de prouver que Bacchis était son esclave. Büchner 1974, p. 370, est aussi d'avis qu'il s'agit seulement d'une menace d'Eschine et que ceci n'est pas une preuve que la jeune fille était libre chez Diphile, car on ne comprend pas pourquoi on paierait alors 20 mines au leno ( Wäre bei Diphilus das Mädchen eine Freie und hätte der Entführer Aussicht, das Mädchen im Prozeß freizubekommen, wäre es kaum das Ziel, dem Kuppler für nichts 20 Minen in die Hände zu geben. 194 ist also eine Drohung »).

32. Rieth 1964, p. 118 sq. En fait, Rieth pense que Térence a changé en respect de Ménandre en introduisant cette variation (qu'on a considérée comme un mensonge nécessaire, 'Notlüge') qui ne corresponde pas au caractère d'Eschine. Je soupçonnerais volontiers que ceci ne se trouve pas chez Ménandre. L'effet en est que même Eschine affiche des défauts après avoir reçu l'éducation de Micion, comme il arrive, selon Aristote, à tous les personnages de la comédie. Mais, alors, penser que Térence a été plus aristotélicien que Ménandre, cela me semble absurde.

33. Büchner 1974.

34. Fantham 1971, p. 993.

35. Pöschl 1975.

36. Johnson 1968.

37. Lord 1977.

38. Gaiser 1967.

39. Rieth 1964, p. 118.

40. Trad. Marouzeau, Térence, III, CUF, p. 159.

41. Je pense que Turpion pouvait ouvrir à Térence l'accès à des recueils de textes de comédies comme celles de Plaute. Sur Ambivius Turpio, voir Garton 1972, p. 62-65 et 236 sq. La question des premières éditions de ces comédies et de possibles interventions des acteurs insérées dans le texte du poète est compliquée, car elle dépend du fait qu'il y ait eu des copies ou des éditions de ces textes, et que l'on puisse les trouver; mais on dispose aujourd'hui du Papyrus d'Herculanum 78, papyrus malheureusement brûlé, mais sur lequel on peut encore lire quelque chose (Carosi 2006). Le texte contenu dans ce papyrus est très probablement l'Obolostates de Caecilius et l'écriture est très ancienne, pas encore complètement normalisée. Sans entrer dans les questions qui touchent à ce texte, cela nous prouve (s'il s'agit vraiment de l'Obolostates de Caecilius, voir les sept fragments dans Guardì 1974, p. 69-71) qu'on avait déjà des copies très anciennes qui pouvaient entrer dans une bibliothèque comme celle des Pisones, qu'il s'agisse de simples copies ou de véritables éditions.

42. Bianco 1962, p. 37 sq.

43. Klose 1966, p. 160 sq.

44. Lentano 1996.

45. Calboli 2002, p. 364. À cet égard je dois me corriger : Lentano 1996 avait parfaitement raison de retrouver Plaute, Mil. 718-722, dans Térence, Ad. 35-38.

46. À cet égard Donat a entrevu que Térence a émis cette affirmation pour prouver qu'il n'avait rien pris de Plaute, mais Donat n'éclaircit pas ce qu'il veut dire. Il est possible qu'il emploie le même critère que nous, mais ce n'est pas sûr. Au contraire on peut affirmer valablement que le grand problème de Térence était de démontrer qu'il n'avait rien pris du tout de Plaute.

47. Il est bien probable qu'il s'agissait de ludi gladiatori, car dans le prologue de l'Hecyre, troisième représentation (v. 40), on dit que la deuxième représentation (aux jeux funèbres de Paul-Émile, 
auxquels avaient été représentés aussi les Adelphes) avait été troublée par les jeux des gladiateurs. Dans ces vers, le prologue dit (v. 38-43) : refero denuo./ primo actu placeo; quom interea rumor uenit / datum iri gladiatores, populus conuolat / tumultuantur clamant, pugnant de loco: / ego interea meum non potui tutari locum. Pour ma part, je pense qu'ici actu - dont c'est la seule occurrence chez Térence - pourrait avoir une valeur générique, mais je crois plutôt que dans cette troisième représentation de l'Hecyre, qui venait après la deuxième (refero denuo) et après les Adelphes, Térence a commencé à effectuer des divisions dans le sens des actes. Donat (p. 200 W.) l'entend comme un des cinq actes, mais Donat venait après Varron. S'agissait-il d'une conséquence des problèmes que Térence avait rencontrés dans les Adelphes au lieu que nous avons traité? C'est une question qui s'ouvre et qui découle de ma solution. On pourrait dater le commencement d'une certaine division en actes chez Térence entre la deuxième et la troisième représentation de l'Hecyre, ou bien à la deuxième, si l'Hecyre a été représentée après les Adelphes, car il ne fallait pas beaucoup de temps pour introduire une indication formelle et primitive des actes, en suivant à peu près la division en xopoũ qu'on trouve dans les papyrus de Ménandre.

48. La première représentation de l'Hecyre avait été troublée par la pugilum gloria (Hec. 33). Si les spectateurs aimaient tellement les pugiles, 'pourquoi ne pas les placer aussi dans ma comédie ?' a peut-être pensé Térence.

\section{RÉSUMÉS}

À partir de modifications apportées par Térence à l'original ménandrien et la manière dont le grammairien Donat en rend compte dans son commentaire, l'article propose trois niveaux d'interprétation de ces modifications du point de vue littéraire: celui du grammairien qui identifie les éléments les plus voyants et indique les éléments du problème; celui des rapports entre Térence et les autres comiques qui ont pu le conduire à des adaptations polémiques; celui enfin de la recherche par Térence, en adaptant les éléments grecs, d'une amplification du caractère moral et éducatif de la comédie ménandrienne.

\section{INDEX}

Index géographique : Athènes

nomsmotscles Aristote, Caecilius Statius, Cicéron, Diphile, Donat, Ennius, Horace, Jérôme, Luscius Lanuvinus, Ménandre, Naevius, Plaute, Porphyrion, Rufin, Térence, Varron, Virgile Mots-clés : comédie grecque, comédie latine, commentaire antique, division en actes et/ou en scènes, emprunt littéraire, grammairiens latins, morale comique, plagiat, traduction

Keywords : Greek comedy, Latin comedy, ancient commentary, Latin grammarians, comic ethics, translation, division into acts and/or scenes, plagiarism, literary imitation and quotation 